\section{Flower Pigments within Hemerocallis fulva L. fm. fulva, fm. rosea, and fm. disticha}

\author{
R.J. Griesbach and L. Batdorf \\ U.S. National Arboretum, Agricultural Research Service, U.S. Department of \\ Agriculture, Beltsville Agricultural Research Center-West, Building 004, \\ Beltsville, MD 20705-2350
}

Additional index words. daylily, flower color, anthocyanin, carotenoid

\begin{abstract}
Various forms of Hemerocallis fulva differed in their relative anthocyanin : carotenoid ratios and the type of anthocyanin present. Hemerocallis fulva fm. fulva contained a single anthocyanin (cyanidin-3-rutinoside) and two carotenoids (zeaxanthin and lutein). Hemerocallis fulva fm. rosea contained a single anthocyanin (cyanidin3-rutinoside) and traces of carotenoids. Hemerocallis fulva fm. disticha contained a single anthocyanin (delphinidin-3-rutinoside) and two carotenoids (zeaxanthin and lutein).
\end{abstract}

Flower colors are due to at least two types of pigments, the carotenoids and flavonoids. The flavonoids can be subdivided into copigments (e.g., flavonols and flavonones) and anthocyanins. In vivo, the anthocyanins and copigments occur as a complex held together through hydrogen bonding (Kondo et al., 1992). The color seen is the result of the physical exchange and interaction of electrons between the carbon rings of the individual anthocyanin and copigment molecules within this complex. The anthocyanin copigment complex is responsible for flower colors red through blue.

Flower colors yellow through orange are due to the presence of carotenoid pigments. Most flowers contain either anthocyanin or carotenoid pigments, not both. When the two pigment classes do occur together, new and unusual colors can be produced (Griesbach, 1984). For example, in orchids, red flowers could be either the result of a red anthocyanincopigment complex and the absence of carotenoids or the result of a magenta anthocyanin-copigment complex and the presence of carotenoids.

Flowers of Hemerocallis fulva contain carotenoid and flavonoid pigments. Three forms of this species exist-fm. fulva with orange flowers [Royal Horticultural Society (RHS) 28 B (RHS, 1966)], fm. rosea with rose flowers (RHS $51 \mathrm{~B}$ ), and fm. disticha with brown flowers (RHS $171 \mathrm{~B}$ ). In this study, we determined the pigments responsible for the several color forms.

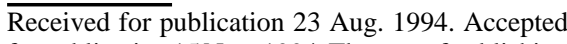
for publication 15 Nov. 1994. The cost of publishing this paper was defrayed in part by the payment of page charges. Under postal regulations, this paper therefore must be hereby marked advertisement solely to indicate this fact.
}

\section{Materials and Methods}

Plant material. Three color forms of $H$. fulva were obtained from the U.S. National Arboretum (USNA), Washington, D.C. Hemerocallis fulva fm. fulva is native to most of Eurasia. In addition, it has a long history of cultivation with many selected clones of unknown origin. The clone of $H$. fulva fm. fulva (USNA accession no. 37426) used in this study was collected in China by S-Y Hu of the Arnold Arboretum of Boston.

Hemerocallis fulva fm. rosea is restricted to a small area near Kuling, China. Arlow Stout of the New York Botanical Gardens selected the clone of $H$. fulva fm. rosea 'Rosalind' (USNA 24421) used in this study. He selected the 'Rosalind' daylily from a group of seedlings derived from selfpollination of a clone collected in 1924 by A.N. Stewart.

Hemerocallis fulva fm. disticha is native to subtropical China and first was collected in 1789. The clone of $H$. fulva fm. disticha (USNA 30365) used in this study was collected in China by M. Matsuoka of the Osaka Botanical Gardens, Japan.

Anthocyanin analysis. The anthocyanin pigments were characterized by high-pressure liquid chromatography (HPLC) as previously described for Petunia hybrida Juss. (Griesbach et al., 1991). The petals and sepals of individual flowers were removed and ground at high speed in a blender (Waring, New Harford, Conn.) with $100 \mathrm{ml}$ aqueous $10 \%$ acetonitrile, $15 \%$ acetic acid, and $1.5 \%$ phosphoric acid. The extract was filtered through no. 3 Whatman paper and reduced to dryness under vacuum at 40C. The residue was then dissolved in 100 $\mu$ liter of the extraction solvent. The anthocyanins were resolved on a $7.8 \times 300-\mathrm{mm}$ Bondapak C18 column (Waters, Milford, Mass.) using a 30-min linear gradient of $0 \%$ to $10 \%$ acetonitrile in aqueous $15 \%$ acetic acid and $1.5 \%$ phosphoric acid, followed by a $10-$ min linear increase to $20 \%$ acetonitrile. Finally, they were held in $20 \%$ acetonitrile for an additional $10 \mathrm{~min}$. The flow rate was $1 \mathrm{ml} \cdot \mathrm{min}^{-1}$, and the eluant was monitored at $540 \mathrm{~nm}$.

The anthocyanins were characterized by coelution, with purified known standards from Hemerocallis (Asen and Arisumi, 1968).

Carotenoid analysis. The carotenoid pigments were characterized by HPLC as previously described for Ornithogalum L. (Griesbach et al., 1993). The petals and sepals of individual flowers were removed and ground in $100 \mathrm{ml}$ acetone and filtered through no. 3 Whatman paper. The solution was extracted three times with petroleum ether using $50 \mathrm{ml}$ each time. The carotenoid-containing ether was reduced to dryness under vacuum, and the residue was dissolved in $100 \mu$ liter $25 \%$ acetonitrile and $75 \%$ methanol. The carotenoids were resolved on a radially compressed, $8 \times$ 100 -mm Bondapak C18 column using a 20min linear gradient of aqueous $75 \%$ to $100 \%$ acetonitrile : methanol (25:75). The solvent then was held at $100 \%$ for an additional 20 $\mathrm{min}$. The flow rate was $1.5 \mathrm{ml} \cdot \mathrm{min}^{-1}$, and the eluant was monitored at $445 \mathrm{~nm}$.

The carotenoids were characterized by coelution with known standards from spinach (Spinacia oleracea L.) (Thammasiri et al., 1986).
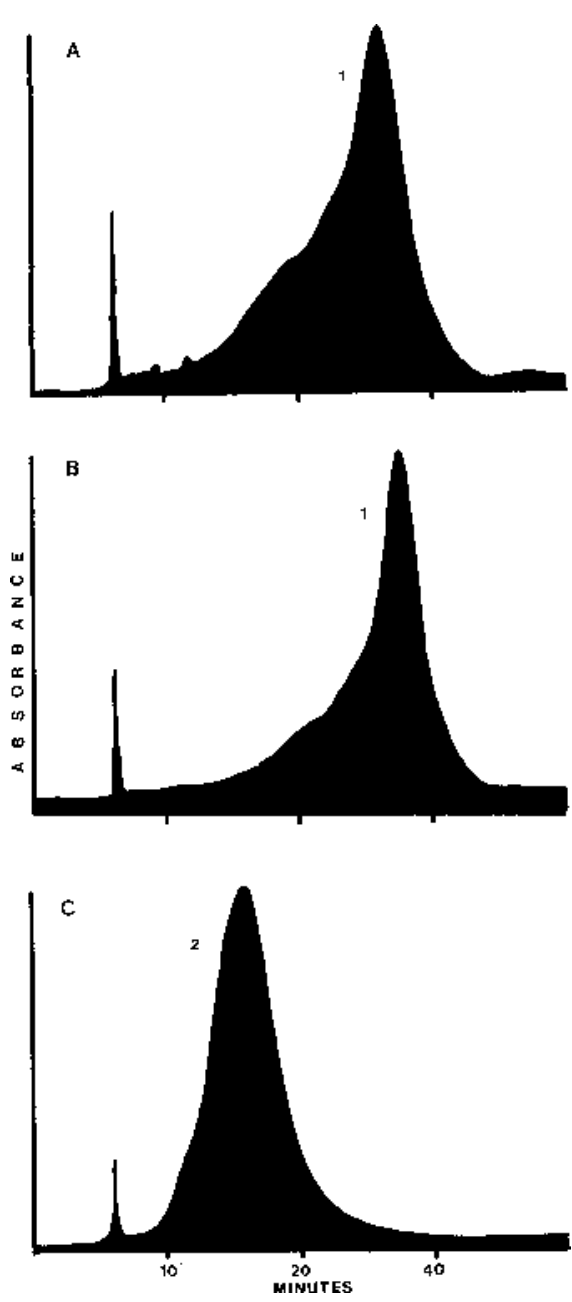

Fig. 1. High-pressure liquid chromatography anthocyanin profiles of Hemerocallis fulva (A) fm. fulva, (B) $\mathrm{fm}$. rosea, and (C) fm. disticha. Cyanidin-3-rutinoside is peak 1 and delphinidin3 -rutinoside is peak 2 . 


\section{Results}

The orange flowers of $H$. fulva fm. fulva contained a single anthocyanin (cyanidin3-rutinoside) (Fig. 1A) and two carotenoids (zeaxanthin and lutein) (Fig. 2A). The rose flowers of $H$. fulva fm. rosea 'Rosalind' contained a single anthocyanin (cyanidin3-rutinoside) (Fig. 1B) and little if any carotenoids (Fig. 2B). The brown flowers of $H$.
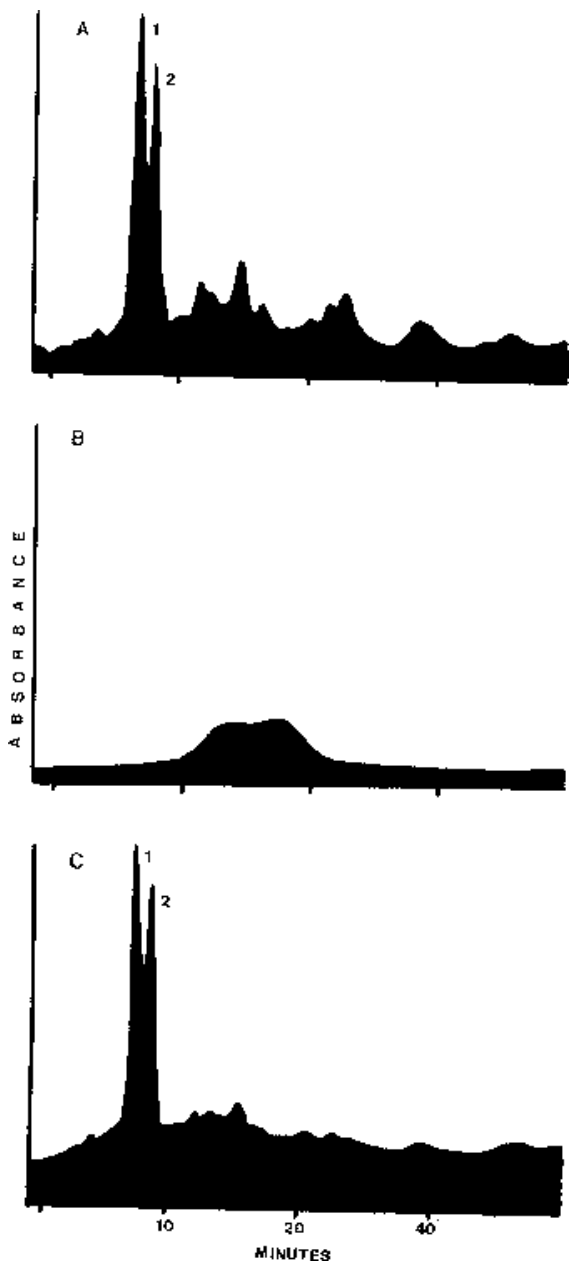

Fig. 2. High-pressure liquid chromatography carotenoid profiles of Hemerocallis fulva (A) fm. fulva, (B) fm. rosea, and (C) fm. disticha. Lutein is peak 1 and zeaxanthin is peak 2 . fulva fm. disticha contained a single anthocyanin (delphinidin-3-rutinoside) (Fig. 1C) and two carotenoids (zeaxanthin and lutein) (Fig. 2C). Hemerocallis fulva fm. rosea differed from the type species, $H$. fulva fm. fulva, because it contained a lower concentration of carotenoid pigments; $H$. fulva fm. disticha differed from the type species because it contained a different anthocyanin pigment.

\section{Discussion}

Hemerocallis breeding has begun only recently. The first artificially created hybrid ('Apricot') was named in 1892. Until 1930, all introduced cultivars were similar to the species progenitors in flower color (Arisumi, 1971). During the 1920s, Stout began a breeding program using new color forms of $H$. fulva. Stout's pioneering breeding program was responsible for creating cultivars with new flower colors (Eddison, 1992). Modern red cultivars contain predominantly cyanidin-3-rutinoside; modern purple cultivars contain predominantly delphinidin (Asen et al., 1968). These are the same pigments found within the various $H$. fulva forms.

We found that $H$. fulva fm. rosea differs from the type species by possessing a reduced carotenoid concentration. This reduced concentration was used by breeders to create pink flowers. Stout (1942) believed that the importance of $H$. fulva fm. rosea in breeding was due to a reduction in plastid pigmentation and reported that the clearest pink colorations were associated with the absence of plastid pigments. Stout (1938) also found that the unique color of $H$. fulva fm. rosea was recessively inherited. He released several pink cultivars, of which the most important to future breeding was 'Charmaine'. This cultivar is in the background of many of the modern pink cultivars.

Hemerocallis fulva $\mathrm{fm}$. disticha differs from the type species by possessing a different anthocyanin (e.g., delphinidin). Although this form was first collected in 1749, it was not recognized formally as a valid botanical entity until recently $(\mathrm{Hu}, 1968)$. After observing the colored photographs and plates in Stout's articles, we believe that he used this form in breeding. Leonian (1939) obtained several of Stout's red cultivars and separated them into two groups, "those possessing purple pigment and those without such a pigment." It seems that $H$. fulva fm. disticha is in the background of Stout's purple-reds. The disticha form most likely is in the background of Stout's most famous cultivar, 'Theron'. 'Theron' expressed a new color that Stout (1938) described as Mars violet. Many of our modern purple and black cultivars are descended from 'Theron'.

Historically, H. fulva fm. fulva contributed gene(s) for red pigmentation, H. fulva fm. rosea for reducing carotenoid pigmentation, and $H$. fulva $\mathrm{fm}$. disticha for purple pigmentation. By mixing these genes in different combinations, the color forms of $H$. fulva are responsible for the wide range of new flower colors (e.g., pink, purple, and red) of the modern Hemerocallis.

\section{Literature Cited}

Arisumi, T. 1971. The changing Hemerocallis. Florist Rev. 147:24-25.

Asen, S. and T. Arisumi, 1968. Anthocyanins from Hemerocallis. Proc. Amer. Soc. Hort. Sci. 92:641-645.

Eddison, S. 1992. A passion for daylilies. Harper Collins, New York.

Griesbach, R.J. 1984. Effects of carotenoid-anthocyanin combinations on flower color. J. Hered. 75:145-147.

Griesbach, R.J., S. Asen, and B. Leonhart. 1991. Petunia hybrida anthocyanins acylated with caffeic acid. Phytochemistry 30:17291731.

Griesbach, R.J., F. Meyer, and H. Koopowitz. 1993. Creation of new flower colors in Ornithogalum via interspecific hybridization. J. Amer. Soc. Hort. Sci. 118:409-414.

Hu, S-Y. 1968. The species of Hemerocallis. Amer. Hort. Mag. 47:86-111.

Kondo, T., et al. 1992. Structural basis of bluecolour development in flower petals from Commelina communis. Nature 358:515-518.

Leonian, L.H. 1939. Adventures in breeding red Hemerocallis. Herbertia 6:202-203.

Royal Horticultural Society. 1966. Royal Horticultural Society colour chart. Royal Hort. Soc., London.

Stout, A.B. 1938. Daylilies with rosy pink coloring. Horticulture 16:226.

Stout, A.B. 1942. Origin and genetics of some classes of red-flowered daylilies. Herbertia 9:161-174.

Thammasiri, K., C.S. Tang, H.Y. Yamamoto, and H. Kamemoto. 1986. Carotenoids and chlorophylls in yellow-flowered Dendrobium species. Lindleyana 1:215-218. 